\title{
Toroidal magnetic fields and associated currents in AGN jets on kiloparsec scales
}

\author{
Sebastian Knuettel* \\ Department of Physics, University College Cork, Ireland \\ E-mail: s.knuettel@mars.ucc.ie \\ Denise C. Gabuzda \\ Department of Physics, University College Cork, Ireland \\ E-mail: d.gabuzda@ucc.ie
}

\begin{abstract}
Helical fields are generally accepted as the magnetic field configuration associated with the launching of astrophysical jets, but it is unclear what role they play on the largest jet scales (if they persist to such distances from the central active galactic nucleus (AGN) ). The synchrotron emission from these jets can be highly linearly polarized, making the behaviour of the polarized radiation a useful tool in examining the magnetic fields of these jets. Observations at multiple wavelengths can be used to construct Faraday rotation measure (RM) images, which provide information about the line-of-sight magnetic field. A significant gradient in the RM transverse to the jet direction may indicate a toroidal or helical magnetic field, which would, in turn, imply the presence of an associated electrical current in the jet. The detection of such gradients on kiloparsec scales can reliably demonstrate that helical or toroidal fields can persist to large distances from the central AGN. We present kiloparsec-scale Faraday rotation maps for NGC 6251, 3C 303 and 3C 219 based on archival data from the Very Large Array. NGC 6251 shows statistically significant transverse RM gradients across its kiloparsec scale jet structure. A previous report of a transverse RM gradient across the jet of $3 \mathrm{C} 303$ proves not to be significant, and we have identified a tentative transverse RM gradient across the jet of 3C 219. The NGC 6251 gradient adds to previous detections of significant transverse Faraday rotation gradients across large-scale jets, which have all corresponded to outward currents. Together with results for AGN jets on parsec scales, which imply inward currents, this suggests that the jets have a current/magnetic field configuration similar to that of a co-axial cable.
\end{abstract}

14th European VLBI Network Symposium Users Meeting (EVN 2018)

8-11 October 2018

Granada, Spain

${ }^{*}$ Speaker. 


\section{Introduction}

\subsection{Magnetic fields and currents in jets}

The radio emission from AGN jets is primarily synchrotron radiation, which is created by relativistic electrons accelerated by magnetic field lines, both of which are found in astrophysical jets. Synchrotron radiation can be highly linearly polarized, up to a theoretical limit of $\sim 70 \%$, making radio polarization measurements a useful probe of the magnetic field properties of jets.

A method to directly infer the existence of toroidal or helical magnetic field in AGN jets is identifying traverse gradients in the Faraday rotation measure (RM) across the jet. Faraday rotation is a change in the observed polarization angle due to the presence of a region of magnetic field and free electrons along the line of sight:

$$
\chi_{\mathrm{obs}}=\chi_{0}+\mathrm{RM} \lambda^{2}, \mathrm{RM} \propto \int_{0}^{1} \mathrm{n}_{\mathrm{e}} \mathbf{B} \cdot \mathrm{dl}
$$

where $\chi_{\mathrm{obs}}$ and $\chi_{0}$ denote the observed and intrinsic polarization angles, $\mathrm{RM}$ is the Faraday rotation measure, $\lambda$ is the observing wavelength, $n_{e}$ is the electron density, $\mathbf{B}$ is the magnetic field and $\mathrm{dl}$ is a unit length vector along the line of sight. This relation is valid when the Faraday rotation occurs outside the emission region; in this case, the measured RM is directly proportional to the line-ofsight magnetic field and electron density. Therefore a significant gradient in the RM transverse to the jet could imply a similarly changing line-of-sight magnetic field component around the jet.

For our analysis, the significance of an RM gradient was calculated using its end-point values and associated errors in RM as follows:

$$
\operatorname{significance}(\sigma)=\frac{\left|\mathrm{RM}_{1}-\mathrm{RM}_{2}\right|}{\sqrt{\Delta \mathrm{RM}_{1}^{2}+\Delta \mathrm{RM}_{2}^{2}}}
$$

Where the RM and $\triangle \mathrm{RM}$ values indicate the rotation measures and associated errors for the start and end points. If a gradient has a significance of $3 \sigma$ or higher and appears relatively smooth and monotonic, it can be considered to be statistically significant and therefore a probable indicator of a toroidal or helical magnetic field component. Such a field should induce an electrical current within the jet according to Ampères law. The statistics show that, on small scales observed with VLBI, a statistically significant majority of transverse RM gradients imply an inward (toward jet base) electrical currents [1], while the gradients detected on larger scales such as those observed with the VLA all infer an outward electrical currents [2].

\subsection{New results}

The new results presented in this publication include multi-wavelength studies of 3 AGN which were previously reported to display transverse RM gradients: NGC 6251, 3C 303 and 3C 219. The uncertainties in the rotation measures and therefore the statistical significance of these gradients was not reported in the original publications, requiring a re-analysis of the data. Furthermore, the analysis of these data can be improved by adding in further, more recent, archival observations to confirm results, as was done with NGC 6251, or to extend the range of wavelengths used, as was done with 3C 303. VLBI observation analysis was not performed for these AGN, 
NGC 6251 being the only one that has been monitored by the MOJAVE project shows little to no polarization at $15 \mathrm{GHz}(<0.5 \%$ polarized as of 2013) therefore a multiwavelegth observation from $1.4-5 \mathrm{GHz}$ to map RM would be unlikely to show detailed polarization structure. Furthermore, no RM map is shown and no median RM value is given in Table 1. of [4] where such a study is performed.

\section{Results and analysis}

\subsection{NGC 6251}

The VLA-scale Faraday-rotation distribution of the $z=0.0234$ AGN NGC 6251 was studied in [5], where the presence of transverse RM gradients was noted. This is a very clear one sided FR I source, with an obviously well collimated jet, although diffuse emission from the counter-jet is visible on larger scales. The collimation was initially believed to be due to a dense X-ray atmosphere surrounding the galaxy, however the X-ray environment is most likely not dense enough to bring this about, making magnetic confinement more likely [6]. We downloaded from the VLA archive the original data at 1.37 and $1.66 \mathrm{GHz}$ observed on 5 December 1980,1.48 GHz observed on 5 November 1979, and $4.9 \mathrm{GHz}$ observed on 5 Nov 1980, published in [5] and calibrated them using standard procedures in AIPS.
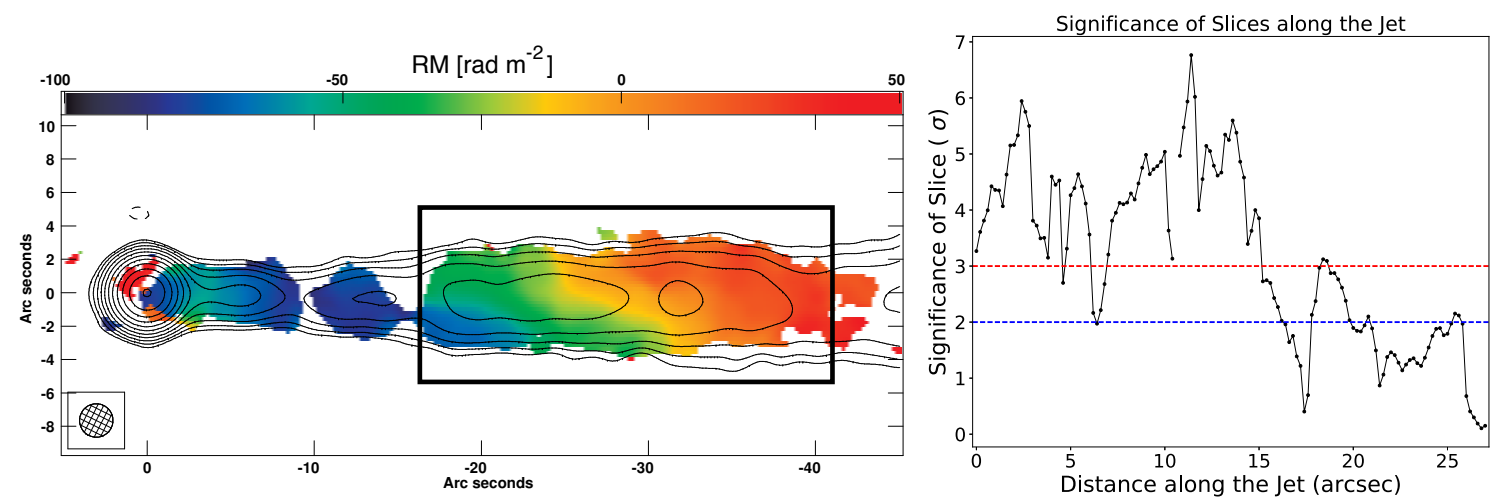

Figure 1: Left panel: Zoomed image of the inner region of NGC 6251 rotated by $-26.5^{\circ}$ showing the RM in the color scale and the Stokes $I$ contours at $1.37 \mathrm{GHz}$ with levels at \pm 0.4 to $409.6 \mathrm{mJy} /$ beam in steps of a factor of two. The $2^{\prime \prime}$ convolving beam is given in the bottom left corner. Right panel: A plot of RM gradient significance versus distance along the jet for the region in the black rectangle in the left panel. A distance of 0 corresponds to the left end of this box. Levels of 2 and $3 \sigma$ are shown as dashed lines in blue and red, respectively.

Figure 1 shows an image of the inner part of the jet rotated by $-26.5^{\circ}$ showing the region of transverse RM gradients. The box shows the region where a series of vertical RM slices were taken and checked for their significance based on Equation 1.2 using automated routines in PYTHON. A plot showing the significance of each slice versus distance along the jet is given on the right. The RM was corrected for Galactic Faraday rotation by subracting the integrated value of $-49 \mathrm{rad} \mathrm{m}^{-2}$ calculated from the diffuse emission in the outer jet of NGC 6251 in [5].

To confirm this result, archival observations at $4.5-6.5 \mathrm{GHz}$ originally taken on 1 December 2013 were downloaded from the VLA archive and calibrated using standard procedures in CASA. 

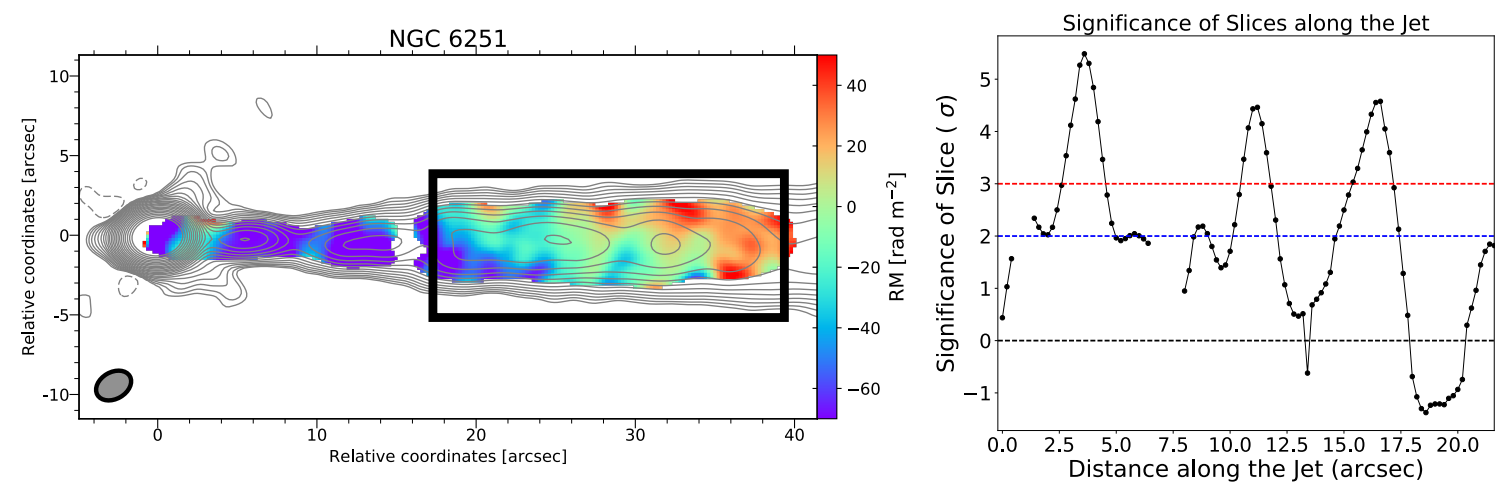

Figure 2: Left panel: Zoomed image of the inner region of NGC 6251 rotated by $-26.5^{\circ}$ showing the RM in the colour scale and the Stokes $I$ contours at $5.5 \mathrm{GHz}$ with levels at \pm 0.075 to $38.4 \mathrm{mJy} /$ beam in steps of factors of $\sqrt{2}$ with the negative contour dashed. The convolving beam of $2 \times 1.5^{\prime \prime}$ with a position angle of $-60^{\circ}$ is given in the bottom left corner. Right panel: Plot of transverse RM gradient significance versus distance along the jet for the region enclosed by the black rectangle. A distance of 0 corresponds to the left of this box. Levels of 0,2 and $3 \sigma$ are shown as dashed lines in black, blue and red, respectively.

Even though these data were not as sensitive to RM due to the smaller range of $\lambda^{2}$, an RM map similar to the map in Figure 1 could be produced. This RM map is displayed in Figure 2 together with a plot of the significances of transverse gradients as a function of distance along the jet for the segment of the jet enclosed by the black rectangle. Although the overall sensitivity to the RM variations is lower than in Figure 1, the significances of the RM gradients go above $3 \sigma$ in some regions, confirming the results shown in Figure 1. Concerning the parsec scale jet for this source, NGC 6251 has been monitored by the MOJAVE ([3]) project and shows little to no polarization at $15 \mathrm{GHz}(<0.5 \%$ polarized as of latest observation in 2013). Multiwavelegth observations to calculate RM are expected to show little or no significant polarization structure, due to depolarization; for example in Table 1. of [4], where such a study is performed, no median RM value is given and no RM map is shown.

\section{$2.23 \mathrm{C} 303$}

A transverse RM gradient across the kiloparsec scale jet of 3C 303 was reported in [7], where the magnitude of the RM gradient is specified, however with no indication of the uncertainties in the RM. We downloaded the original data for 1.4 and $4.9 \mathrm{GHz}$ observed on the 20 April 1981 from the VLA archive and calibrated them using AIPS. To extend the range of wavelengths, an archival observation at $15 \mathrm{GHz}$ observed on 8 December 1986, at a similar resolution, was also downloaded from the archive and calibrated. As the rotation measure image produced in [7] was made using only two frequencies $(1.4 \mathrm{GHz}$ and $4.9 \mathrm{GHz})$, adding the $15 \mathrm{GHz}$ data gives a better insight into the errors in the $\chi$ vs $\lambda^{2}$ fits and therefore a better estimate of the error in the RM. The RM image was also corrected for Galactic Faraday rotation by having the integrated value of $18 \pm 2 \mathrm{rad} \mathrm{m}^{-2}$ [8] subtracted from the calculated RM, as was done in [7]. The RM map is presented in Figure 3 with a zoomed in region of the knot labelled 'E3' in [7], where the purported transverse RM gradient was first reported. A gradient is visible by eye across this knot at the location of the black line through E3 in Figure 3. Applying the same method which was used for the region with gradients 


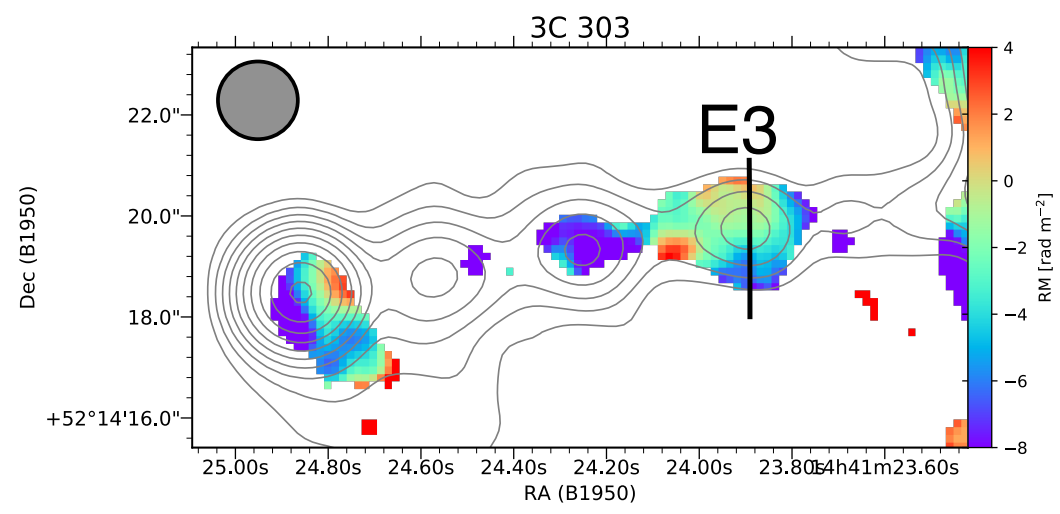

Figure 3: A zoomed in image showing the core and knotty inner jet of 3C 303. The RM is given in the colour scale and the Stokes $1.4 \mathrm{GHz} I$ contours run from 4 to $256 \mathrm{mJy} /$ beam in steps of factors of $\sqrt{2}$. The location of the previously claimed gradient is marked by the black vertical line. The convolving beam of $1.5^{\prime \prime}$ is displayed in the top left corner of the panel.

in NGC 6251, possible transverse RM gradients in the 'E3' knot region were analysed and tested for their significance. The significances of these gradients were not more then $1.5 \sigma$, and therefore we conclude that this seeming transverse RM gradient most likely came about by chance. There is little published about the parsec scale jet; a one-sided jet is seen in the $5 \mathrm{GHz}$ global VLBI image in Figure 15. of [9], but no additional polarization information on these scales is known to the authors.

\section{$2.33 \mathrm{C} 219$}
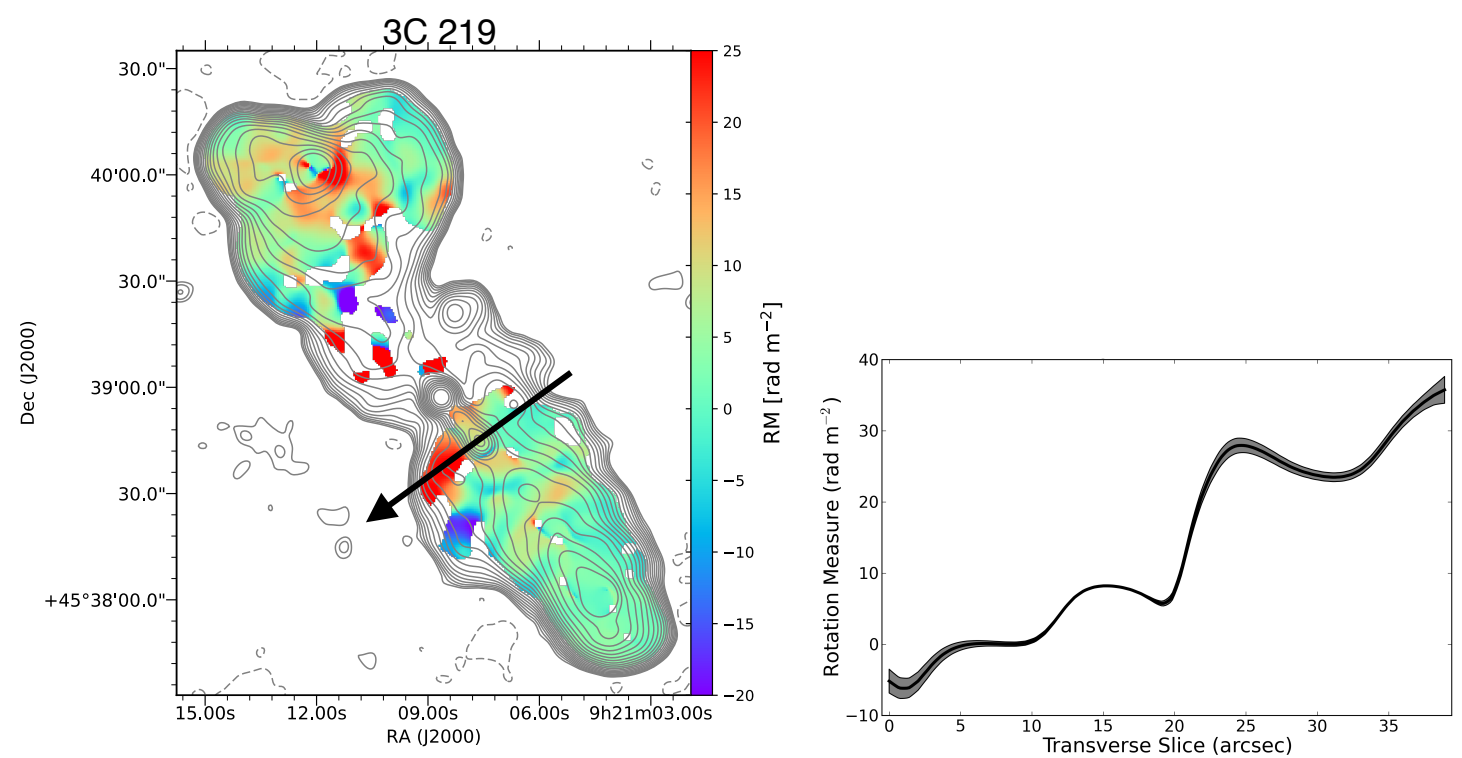

Figure 4: Left panel: Image of 3C 219 showing the RM in the colour scale and the Stokes $I$ contours at $4.9 \mathrm{GHz}$ with levels of \pm 0.075 to $38.4 \mathrm{mJy} /$ beam in steps of factors of $\sqrt{2}$ with the negative contour dashed. The arrow traces the direction of the previously reported RM gradient. Right panel: A plot of RM versus transverse jet distance plotted corresponding to the black arrow in the left panel. 
This is a twin lobed radio galaxy at redshift $z=0.1745$, for which a possible RM gradient was reported in [10]. The presence of a helical magnetic field threading the jet was not ruled out in [10], however this was not the focus of that paper, and the possible transverse RM gradient was not fully analysed. The raw data at 1.3, 1.6 and $4.9 \mathrm{GHz}$ observed on 6 September 1986 used in [10] were downloaded from the VLA archive and calibrated using CASA. The RM map was made using a common convolving beam of $5^{\prime \prime}$ and is shown in Figure 4. A Galactic contribution to the $\mathrm{RM}$ of $-12.6 \mathrm{rad} \mathrm{m}^{-2}$ [11] was subtracted. Our analysis showed that the RM profiles across the jet in the region where possible transverse RM gradients were claimed in [10] are generally not monotonic. A slice of RM values in the region of transverse RM gradients reported in [10] taken at the position shown in the left panel is given in the right panel as an example. The slice is not monotonic, but there is nevertheless a clear trend in the RM across the jet; the difference in the end RM values is highly significant ( $\sim 14 \sigma$ ). In addition, the sign of the RM changes from one end of the slice to the other, indicating a change in the line-of-sight magnetic field component, and this cannot be explained by a change in the electron density alone. Some random fluctuations in the RM structure due to local patchiness and turbulence are not unexpected, and it is possible that we are seeing the result of a transverse RM gradient due to a toroidal field component with a random RM distribution superposed. The rest of the RM distribution seems fairly patchy. All considered, we regard this only as a tentative transverse RM gradient, particularly given that it is localized in only a very small region of the jet. Analysis of observations on similar scales with higher sensitivity to Faraday rotation may help resolve this question. Images of the parsec scale jet and analysis of VLBI data are not published to the best of our knowledge. The unresolved core of this AGN has a $5 \mathrm{GHz}$ flux of $<70 \mathrm{mJy}$ in the data published here, hence a high-resolution VLBI detection with significant polarization information would be unlikely.

\section{Conclusion}

We have presented VLA Faraday rotation images for 3 AGNs. Data for NGC 6251 from archival observations at 1.37, 1.48, 1.66 and $4.9 \mathrm{GHz}$ taken between 1979 and 1980 have been re-analysed, as well as previously unpublished wideband observations at $4.5-6.5 \mathrm{GHz}$ taken in December 2013. Both of these datasets show statistically significant RM gradients across the kiloparsec-scale jet of NGC 6251, confirming the suspicions of Perley et al [5].

The previously reported kiloparsec scale transverse RM gradient across the jet of 3C 303 has been shown not to be significant by our analysis, which did not indicate the presence of any transverse RM gradients with significance $>1.5 \sigma$.

Our analysis of a transverse RM gradient in 3C 219 previously noted in [10] yielded only a tentative result: only a small portion of the jet shows a possible transverse gradient, which may be quite significant but is not monotonic.

Looking at the total collection of examples of statistically significant large-scale transverse RM gradients in AGN jets, there are now 12 results (previous results from [12] and [2] and the result for NGC 6251 from this publication). The direction of all these gradients imply outward electrical currents. Using a binomial probability distribution this implies a strong preference for outward currents and an only $0.024 \%$ chance of being a random coincidence. Including the tentative result for 3C 219, which has a gradient inferring an inward current, and again applying the binomial proba- 
bility distribution, the chance that the outward current preference is random increases only slightly to $0.16 \%$. This conclusively demonstrates a strong preference for the dominant currents on kiloparsec scales to be outward. When combined with the statistically significance preference for the dominant currents on parsec scales to be inward, this suggests a co-axial cable-like configuration of B fields and currents, as suggested earlier in $[1,12]$.

Acknowledgements: This research has been funded by the Irish Research Council under the Government of Ireland Postgraduate Programme. The VLA is an instrument of the National Radio Astronomy Observatory, a facility of the National Science Foundation operated under co-operative agreement by Associated Universities, Inc.

\section{References}

[1] D. C. Gabuzda, M. Nagle, N. Roche, The Jets of AGN as giant co-axial cables, A\&A 612 (2017) A67 [ArXiv:1712.08414]

[2] S. Knuettel, D. Gabuzda, S. P. O’Sullivan, Evidence for Toroidal B-Field Components in AGN Jets on Kiloparsec Scales, Galaxies 5 (2017) 61

[3] M. L. Lister, M. F. Aller, H. D. Aller, M. A. Hodge, D. C. Homan, Y. Y. Kovalev, A. B. Pushkarev, T. Savolainen MOJAVE. XV. VLBA 15 GHz Total Intensity and Polarization Maps of 437 Parsec-scale AGN Jets from 1996 to 2017 ApJS 234 (2018) 12

[4] T. Hovatta, M. L. Lister, M. F. Aller, H. D. Aller, D. C. Homan, Y. Y. Kovalev, A. B. Pushkarev, T. Savolainen MOJAVE: Monitoring of Jets in Active Galactic Nuclei with VLBA Experiments. VIII. Faraday Rotation in Parsec-scale AGN Jets, AJ 144 (2012) 105

[5] R. A. Perley, A. H. Bridle, A. G. Willis, High-resolution VLA observations of the radio jet in NGC 6251, ApJS 54 (1984) 291-334

[6] K.-H. Mack, J. Kerp, U. Klein, The X-ray jet and halo of NGC6251, A\&A 324 (1997) 870-887

[7] P. P. Kronberg, R. V. E. Lovelace, G. Lapenta, S. A. Colgate, Measurement of the electric current in a kpc-scale jet, ApJ 741 (2011) L15 [ArXiv:1106.1397]

[8] M. Simard-Normandin, P. P. Kronberg, S. Button, The Faraday rotation measures of extragalactic radio sources, ApJS 45 (1981) 97-111

[9] G. Giovannini, W. D. Cotton, L. Ferreti, L. Lara, T. Venturi, VLBI Observations of a Complete Sample of Radio Galaxies: 10 Years Later, ApJ 5522001 508-526

[10] D. A. Clarke, A. H. Bridle, J. O. Burns, R. A. Perley, M. L. Norman, Origin of the structures and polarization in the classical double 3C 219, ApJ 385 (1992) 173-187

[11] A. R. Taylor, J. M. Stil, C. Sunstrum, A Rotation Measure Image of the Sky, ApJ 702 (2009) 1230-1236

[12] D. M. Christodoulou, D. C. Gabuzda, S. Knuettel, I. Contopoulos, D. Kazanas, C. P. Coughlan, Dominance of outflowing electric currents on decaparsec to kiloparsec scales in extragalactic jets, A\&A 591 (2016) A61 [ArXiv:1605.08991] 